\title{
Hemorragia uterina disfuncional
}

\author{
Luis Ernesto Pérez Agudelo*
}

RESUMEN: La hemorragia uterina disfuncional (HUD) constituye el $75 \%$ de la hemorragia uterina anormal (HUA). EI otro $25 \%$ es hemorragia uterina bien sea por patología del tracto reproductivo, patología sistémica, o iatrogénica. El diagnóstico de HUD sólo se puede sustentar cuando no se han encontrado causas orgánicas después de una cuidadosa historia clínica, examen físico, pruebas de laboratorio acordes, ecografía transvaginal, y en muchos casos biopsia endometrial e histeroscopia. Las mujeres adolescentes por inmadurez del eje endocrino, y las climatéricas por involución son las más susceptibles de presentar HUD. EI episodio agudo es mejor controlado con estrógenos a altas dosis, o con raspado uterino en caso de hipovolemia. El tratamiento crónico, para evitar recurrencias, depende de las necesidades de cada paciente: progestágenos cíclicos, anticonceptivos orales de microdosis, estrógenosprogestágenos, inductores de la ovulación, ablación histeroscópica, e histerectomía.

PALABRAS CLAVES: Hemorragia uterina anormal (HUA), hemorragia uterina disfuncional (HUD), hemorragia uterina orgánica, adolescencia, climaterio.

SUMMARY: Dysfunctional uterine bleeding (DUB) represents $75 \%$ of abnormal uterine bleeding (AUB). Only $25 \%$ of AUB including organic causes that may be subdivided into reproductive tract disease, systemic disease, and iatrogenic causes. When an organic cause of AUB cannot be found by careful history and physical examination, adjuntive diagnostic tests, transvaginal ultrasonography, endometrial biopsy, and hysteroscopy when necessary: then by exclusion on the diagnosis is of DUB is assumed. Women with a high-risk of present DUB are adolescents and climaterics because inmadurity and involution of the endocrine axis respectively. The acute bleeding episode is best controlled with the use of high-dose estrogen, or with an uterine curettage for patients with bleeding resulting in hypovolemia. The cronic management is determined by the needs of the patient: Oral cyclic progestins, oral microdoses contraceptives, oral estrogens-progestins, clomiphene citrate, endometrial hysteroscopic ablation, and hysterectomy.

KEY WORDS: Abnormal uterine bleeding (AUB), dysfunctional uterine bleeding (DUB), organic uterine bleeding, adolescent, climateric.

\section{Tabla 1}

HEMORRAGIA UTERINA ANORMAL

Cualquier sangrado proveniente del útero diferente del menstrual, es anormal. La hemorragia uterina anormal (HUA) puede ser: 1) Hemorragia uterina orgánica: ginecológica, sistémica, o iatrogénica, y 2) Hemorragia uterina disfuncional (HUD) (tabla 1) (1-3).

Hemorragia uterina disfuncional (HUD) es la hemorragia anormal proveniente de la cavidad uterina en ausencia de lesiones orgánicas detectables. Aunque se diagnostica por exclusión de las causas orgánicas, es importante tenerla en cuenta porque constituye el $75 \%$ de las hemorragias uterinas anormales. Es ocasionada por alteraciones disfuncionales del eje endocrino HipotálamoHipófisis-Ovario-Endometrio. Ocurre el $25 \%$ de las veces en la pubertad, $25 \%$ en la edad adulta reproductiva, y $50 \%$ en el climaterio.

Ginecólogo Obstetra. Jefe Unidad Fertilidad Hospital Militar de Bogotá
Causas orgánicas (25\%)

a) Enfermedades del tracto reproductivo

- Embarazo: Aborto, ectópico, enfermedad trofoblástica.

- Infecciones: Cervicitis, endometritis, salpingitis.

- Benignas Ulceras vulvares, carúncula uretral, ectropión, pólipo, miomatosis, endometriosis, adenomiosis.

- Malignas: Cáncer vaginal, cervical, uterino, ovárico.

- Trauma: Cuerpo extraño, DIU, o trauma sexual.

b) Enfermedad sistémica

- Endocrinas: Hipo o hipertiroidismo, hiperprolactinemia, lesiones hipotalámica, hipofisiarias, ováricas, y suprarrenales.

- Coagulopatías: Enfermedad de Von Willebrand, púrpura trombocitopénica idiopática, anemia, leucemia, linfomas, hemofilia.

- Trastornos hepáticos y renales.

c) Causas iatrogénicas:

Estrógenos, anticonceptivos orales, corticoides, andrógenos, danazol, espironolactona, anabolizantes, anticolinérgicos, bloqueadores hipotalámicos, digitálicos, y anticoagulantes.

Hemorragia uterina disfuncional $(75 \%)$ 


\section{Fisiopatología}

El funcionamiento normal del sistema reproductor femenino está determinado por el funcionamiento endocrino coordinado de tres estaciones de relevo: núcleo arcuato del hipotálamo, gonadotropo hipofisiario, y folículo ovárico. La acción de las mismas se refleja en el endometrio, con el establecimiento de los ciclos menstruales y la reproducción. La menarquia, entre los 8 y los 14 años, es el punto de partida; se espera una completa maduración del sistema hacia los 18; un funcionamiento maduro y normal entre los 18 y los 40; y la involución entre los 40 y los 60 años.

En plena madurez funcional, la magnitud de la respuesta del gonadotropo a la GnRH producida por el núcleo arcuato, es modulada por el estradiol folicular como retroalimentación negativa durante la fase folicular del ciclo menstrual. Una vez adquirida la dominancia folicular, si la producción de estradiol excede los $150 \mathrm{pg} /$ $\mathrm{ml}$ y por un mínimo de 36 horas, se ejerce una retroalimentación positiva sobre el gonadotropo para la producción de los picos ovulatorios de las gonadotropinas y el estímulo inicial en el funcionamiento del cuerpo lúteo.

En cada ciclo el endometrio parte de su estrato basal de reserva, los estrógenos hacen proliferar sus glándulas y vasos y formar receptores a la progesterona. En la fase lútea sus glándulas se vuelven tortuosas y el estroma se compacta; y en fase de implantación está listo para recibir y prodigar vida al embrión en sus primeros días. En ausencia de embarazo, sin hCG, sobreviene la declinación funcional del cuerpo lúteo, con la consiguiente deprivación de estrógenos y progestágenos produciéndose vasoconstricción rítmica de las arterias espirales, isquemia tisular, estasis, y desprendimiento de la capa basal endometrial.

Los ciclos menstruales ovulatorios normales se caracterizan por tener una periodicidad de $28 \pm 7$ días, un sangrado menstrual de $60 \pm 20 \mathrm{ml}$, y una duración de la menstruación de $4 \pm 2$ días.

Las alteraciones con frecuencia, cantidad y duración del sangrado menstrual, nos permiten clasificar semiológicamente los tipos de sangrados, ya que son los motivos de consulta (tabla 2).

La HUD es la consecuencia de alteraciones disfuncionales del eje endocrino Hipotálamo-HipófisisOvario-Endometrio. Por eso es frecuente durante la adolescencia $(25 \%)$ por inmadurez neuroendocrina reproductiva; y especialmente durante el climaterio (50\%) por involución. Consecuentemente el $85 \%$ de las mujeres con HUD son anovulatorios. La proliferación endometrial estimulada por estrógenos sin oposición de progestágenos llevan a la hiperplasia con excesiva vascularización sin vasoconstricción cíclica, favoreciendo la hemorragia al final de las oligomenorreas. En el climaterio tardío (cerca de la menopausia) son frecuentes las polimenorreas con sangrados más frecuentes e intermitentes, por la presencia de folículos viejos y refractarios a las gonadotropinas. En ellas se verifica disminución del estradiol y aumento progresivo de la FSH (4).

Además de la inmadurez y la involución del eje endocrino, la HUD es causada por Disfunción Hipotálamo-
Tabla 2

PATRONES DE SANGRADO UTERINO ANORMAL

\begin{tabular}{|ll|}
\hline Amenorrea & Ausencia de menstruación a los 3 meses \\
Oligomenorrea & Intervalos mayores de 35 días \\
Polimenorreas & Intervalos mayores de 21 días \\
Menorragia & Hemorragia mayor de $80 \mathrm{ml}$, o de 6 días \\
Hipermenorrea & Sinónimo de monorragia \\
Metrorragia & Hemorragia con intervalos irregulares \\
\hline
\end{tabular}

Hipofisiaria ocasionada por estrés, cambios abruptos de peso, y ejercicios físicos intensos; en estos casos las betaEndorfinas aumentadas alteran la ciclicidad y pulsatilidad de la GnRH.

Sólo un 15\% de mujeres con HUD son ovulatorias; pero son ciclos ovulatorios anormales, con alteraciones en la respuesta folicular a las gonadotropinas y por lo tanto con niveles subnormales de estradiol y de progesterona. El manchado mesocíclico es debido a pequeñas caídas del estradiol. Cuando hay síndrome LUF, el quiste luteínico conduce a hipermenorreas seguidas de algunas semanas de amenorrea.

Al final la hemorragia ocurre por cuatro mecanismos:

1. Deprivación estrogénica: los estrógenos sin oposición de progesterona, en ciclos anovulatorios, pueden llevar el endometrio a la hiperplasia, al elevarse bajan las gonadotropinas y caen ellos mismos fallando la vasodilatación endometrial y produciéndose ulceraciones irregulares y hemorragia, la cual aumenta por la acción de las prostaglandinas, y por la actividad fibrinolítica endometrial. También es el mecanismo mesocíclico ovulatorio y cuando se descontinua el tratamiento con estrógenos.

2. Trans-estrogénica: bajas concentraciones de estrógenos fallan en su mecanismo proliferativo endometrial y causan hemorragia.

3. Deprivación de progesterona: cuando hay mal funcionamiento del cuerpo lúteo.

4. Tras-progestacional: ocurre cuando las concentraciones de progesterona son superiores a las de estrógenos, o sea un radio estrógenos/progesterona desfavorable. Es frecuente durante el tratamiento oral o parenteral de progestágenos.

\section{Diagnóstico}

La HUD se diagnostica solo una vez que se hayan excluido las otras causas de HUA (Tabla 1).

En niñas es preciso descartar: procesos infecciosos inflamatorios, trauma, cuerpos extraños, causas urológicas, neoplasias, pubertad precoz, y causas iatrogénicas (5).

En adolescentes se debe pensar primero en trastornos hematológicos (20-50\%): Von Willebrand, otras coagulopatías, leucemia, púrpura trombocitopénica e idiopática, y enfermedades hepato-renales (6-8).

En edad reproductiva son trastornos del embarazo, hasta que no se pruebe lo contrario. 
En climatéricas (perimenopausia y postmenopausia) se deben descartar primero: adenocarcinoma (20\%), hiperplasia endometrial (20\%), y pólipos endometriales (25\%) (9).

Una historia clínica detallada, el cuidadoso examen físico, el laboratorio y algunos paraclínicos nos ayudan a hacer el diagnóstico diferencial.

Laboratorio: Cuadro hemático completo, test de embarazo, citología cervical, TSH, PRL, FSH, T, DHEA-S, perfil de coagulación (plaquetas, tiempo de coagulación, PT, PTTa), y Progesterona mesuloteínica, según el caso.

Biopsia endometrial: una vez excluida la posibilidad de embarazo o de enfermedad pélvica inflamatoria: En toda paciente mayor de 35 años; y en menores de 35 sólo si son obesas, diabéticas o hipertensas por la posibilidad de hiperplasia o CA endometrial.

Ecografía: Si existe la sospecha de miomatosis, adenomiosis, patología ovárica, aborto o embarazo ectópico. La transvaginal tiene sensibilidad del 60-88\% y especificidad del $68-90 \%$ para patologías intracavitarias (9).

Histerosonografía: Ante la posibilidad de patología tumoral con invasión de la cavidad uterina.

Histeroscopia: Con los histeroscopios de diámetro pequeño $(5 \mathrm{~mm})$, no se requiere dilatación cervical, ni anestesia general, y se pueden usar a nivel de consultorio. Ibuprofen $600 \mathrm{mg}$ y Lidocaína paracervical al 1\%, son suficientes. Se diagnostican patologías intracavitarias tales como miomas submucosos e intramurales, pólipos, hiperplasia endometrial, CA focalizado, etc. Sensibilidad del 79\%, y especificidad del 93\% (10-12).

La figura 1 muestra un flujograma de diagnóstico diferencial, que podemos seguir en la práctica.

\section{Tratamiento}

Una vez hecho el diagnóstico diferencial con patología orgánica, el tratamiento de la HUD se divide en dos partes:

Inmediato para suprimir el sangrado, y mediato para prevenir las recurrencias (fig. 2). Siempre se tendrá como objetivos del tratamiento controlar el sangrado, evitar las recurrencias, preservar la fertilidad, corregir los desórdenes asociados, e inducir la ovulación en mujeres que deseen concebir.

Tratamiento inmediato. A buen juicio del médico se pueden elegir cuatro modalidades:

1) Estrógenos conjugados a altas dosis para promover rápido recrecimiento endometrial y recubrir áreas desprovistas de epitelio, seguidos de progestágenos. Para muchos es el tratamiento de elección. Estrógenos conjugados equinos (Ayerogen, Premarin) $25 \mathrm{mg}$ IV cada 4 horas, o si se prefiere $2.5 \mathrm{mg}$ oralmente cada 4 horas hasta que la hemorragia ceda (24-48 hrs). Continuar $1.25 \mathrm{mg}$ orales por 3 semanas, agregando progestágenos las 2 últimas semanas. Es lo más recomendado en tres situaciones: a) Si la hemorragia ha sido prolongada, b) Si se obtiene escasa cantidad de tejido al realizar la biopsia, y c) Si la paciente estaba en tratamiento con progestágenos y continúa sangrando (13-14).
Figura 1

\section{FLUJOGRAMA DIAGNOSTICO (HUA)}

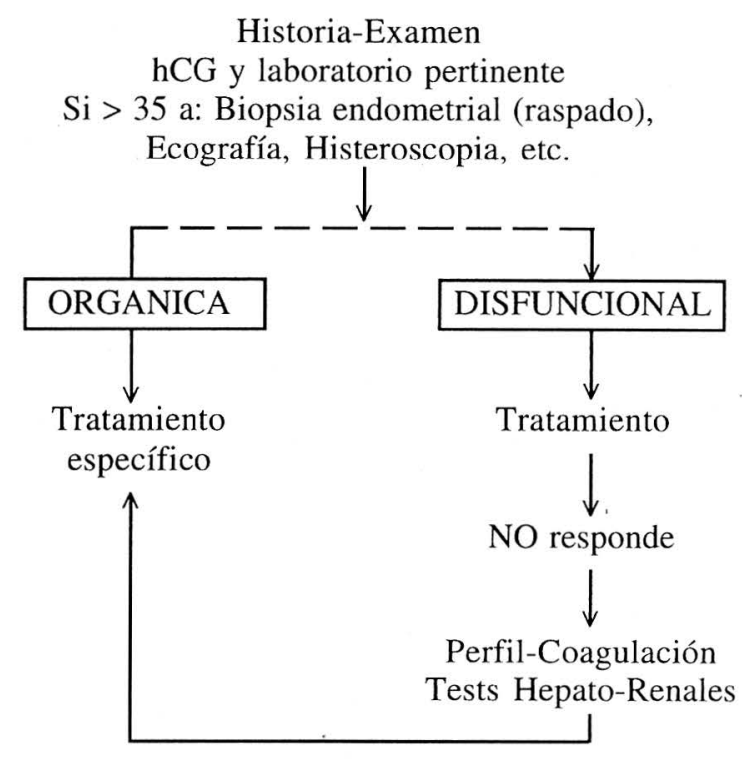

2) Raspado-biopsia si la hemorragia es profusa, porque la paciente gasta más de una toalla higiénica/hora y el hematocrito es menor de 30 . Se debe seguir con un ciclo de estrógenos-progestágenos.

3) Anticonceptivos orales de microdosis, una tableta cuatro veces al día por una semana.

4) Progestágenos por 12 días, si se supone que ha habido un efecto estrogénico proliferativo, y si no se conoce el resultado de la biopsia endometrial, si necesaria. Medroxiprogesterona (MXP), (Provera) 10 a $20 \mathrm{mg} /$ día. También Acetato de noretisterona (Primolut-nor) 10 a $20 \mathrm{mg} /$ día.

Una vez terminado el tratamiento inmediato vendrá una hemorragia por deprivación, y entonces se dará el tratamiento para evitar recurrencias.

Tratamiento preventivo. Ya conociendo los resultados de la biopsia, ecografía, hormonas, histeroscopia, etc, se puede corroborar que se trata de HUD y entonces para el tratamiento preventivo existen varias alternativas hormonales por 3 ciclos, o cirugía, acordes con la edad y necesidades de cada paciente:

1) Progestágenos para adultos y adolescentes que sólo quieran regular el ciclo. MXP $10 \mathrm{mg} / \mathrm{día}$ del 13 al $25 \mathrm{de}$ cada ciclo. También Noretindrona $15 \mathrm{mg} /$ día.

2) Anovulatorios orales de microdosis para quienes quiera además planificar (15). Usados en pacientes entre los 40 y 50 años de edad, hoy en día se consideran de elección (sin el miedo que producían los de macrodosis) porque además de evitarles un embarazo no deseado, las protegen contra la patología benigna del seno y del endometrio, y reducen el riesgo del CA ovárico y endometrial (16). Además les quitan los síntomas del hipoestrenismo y les minimizan los riesgos de la osteoporosis (17). DIUs con progestágenos producen reducción del $97 \%$ del sangrado al año de uso. 
Figura 2

TRATAMIENTO DE LA HUD

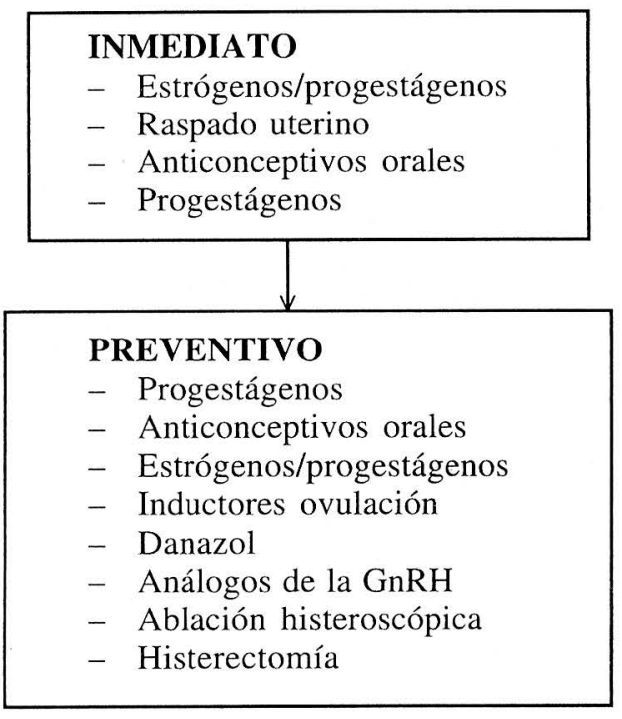

Los AINES se asocian a los progestágenos, y a los anovulatorios orales para las HUD ovulatorias que no responden bien por el imbalance de prostaglandinas que generalmente presentan. Se administran durante los días de sangrado, o por los 3 primeros días de la menstruación.

3) Estrógenos-Progestágenos en forma cíclica para climatéricas, con contraindicaciones para anovulatorios (fumadoras, enf. vascular). Ayerogen 0.625-1.25 mg/d por 25 días, más Provera $10 \mathrm{mg} / \mathrm{d}$ del 13 al 25 día.

4) Inductores ovulatorios para pacientes que deseen embarazo.

5) Danazol $200 \mathrm{mg} /$ día por 3 ciclos ha mostrado ser más útil que la noretindrona $15 \mathrm{mg} /$ día (18). A dosis de $400 \mathrm{mg} /$ día es especialmente útil, como coadyuvante, en casos de hemorragia secundaria a anemia aplástica, púrpura trombocitopénica idiopática, hemofilia, y enfermedad de Christmas (19).

7) Análogos de la GnRH. Utiles también como coadyuvantes pre y durante el tratamiento de pacientes con trastornos de coagulación, en trasplantes de médula ósea, o que reciben quimioterapia, y que han fallado a otros medicamentos. Se han logrado amenorreas en el $77 \%$ administrando Nafarelina IN, o Luprolide IV 1 mg/ día (20).

8) Ablación endometrial histeroscópica con empleo de LASER (Nd-Yag), resectoscopio (electrodiatermia), o radiofrecuencia. Previamente se produce atrofia endometrial con GnRHa, Danazol $600 \mathrm{mg} / \mathrm{d}$, o Medroxiprogesterona oral, por 2 meses. Se practica en pacientes que no responden al tratamiento hormonal, que no desean más hijos, y en quienes la histerectomía está contraindicada. Acorde a la experiencia del cirujano se logra que el 73-100\% queden en amenorrea o hipomenorrea (21-22).

9) Histerectomía como último recurso, después de revisar el diagnóstico diferencial y la terapia médica adecuada.

\section{BIBLIOGRAFIA}

1. Brenner PF. Differencial diagnosis of abnormal uterine bleeding. Am. J. Obstet. Gynecol. 1996; 175: 766-799.

2. Speroff L. Hemorragia uterina anormal. En: Benzon RC. Diagnóstico y tratamiento ginecobstétrico 2d. edición; México, Editorial El Manual Moderno, 1982; 102-126.

3. Pérez LE., Camargo F. Hemorragia Uterina Disfuncional. En: Pérez LE. Infertilidad y endocrinología reproductiva; primera edición; Bogotá, Gamacolor Editorial, 1991; 183-188.

4. Awwad JT., Toth TL., Schiff I. Abnormal uterine bleeding in the perimenopause. International $\mathbf{J}$ of Fertility and Menopausal Studies 1993; 38(5): 261-269

5. Hill MCW., Oppenheimer LW., Morton KE. The etiology of vaginal bleeding in children. A 20 years review. Br. J. Obst. Gynaecol. 1989; 96: $467-470$

6. Claessens EA., Cowell CA. Acute adolescent menorrhagia. Am. J. Obstet. Gynaecol. 1981; 139: 277-280.

7. Consejo Editorial. Hemorragia genital anormal de origen disfuncional -HUD- en adolescentes. Controversias en Obstetricia y Ginecología 1996; 1(3): 22-295.

8. Bruce M., Ewenstein MD. The pathophysiology of bleeding disorders as abnormal uterine bleeding. Am. J. Obstet. Gynecol. 1996; 175: 770-777.

9. Dijkhuizen FP., Brolmann $\mathrm{H}$ et al. La exactitud de la ultrasonografía transvaginal en el diagnóstico de las anormalidades endometriales. Rev. Col. Obstet. Gin. 1997; 47(2): 133-134.

10. Towin N., Gviazda I., March CM. Histeroscopia en el consultorio versus ultrasonografía transvaginal en la evaluación de pacientes con hemorragia uterina excesiva. Rev. Col. Obs. Gin. 1996; 47(3): 211.

11. Loffer FD. Hysteroscopy with selective endometrial sampling compared with D\&C for abnormal uterine bleeding: the value of a negative hysteroscopic view. Obstet. Gynecol. 1989; 73: 16-20.
12. Labastida R., Ubeda A., Carach M., Penella J. Cinco mil cincuenta y nueve (5.059) histeroscopias diagnósticas: método, indicaciones y resultados. Progresos de Obst. y Ginecol 1994; 37: 348-354.

13. Dawood MY., Ravnikar VA., Schneider G., Warren MP. Managing dysfuncional uterina bleeding. Patient Care 1992; 15: 2-7.

14. Chuong CJ., Brenner PF. Management of abnormal uterine bleeding. Am. J. Obstet. Gynecol. 1996; 175: 787-792.

15. Lin MC., Gosink BB., Wolf SI et al. Endometrial thickeness after menopause: Effects of hormonal replacement. Radiology 1991; 180: 427.

16. Mishell DR Jr. Contraception. N Engl J Med. 1989; 320: 777.

17. Kleerekoper M., Brienza RS., Shultz LR., Johnson CC. Oral contraceptive use may protect against low bone mass. Arch. Int. Med. 1991; 151: 197.

18. Higham JM., Shaw RW. A comparative study of danazol, a regimen of decreasing doses of danazol, and norethindrone in the treatment of objetively proven unexplained menorrhagia. Am. J. Obstet. Gynecol. 1993; 169(5): 1134-1139.

19. Nakamura G., Yoshimitsu K., Ootsurka $\mathbf{H}$ et al. Long term danazol treatment for an adolescent girl with aplastic anemia. Asia Oceani J. Obstet. Gynecol. 1986; 12: 365-370.

20. Laufer MR., Rein MS. Tratamiento de la hemorragia uterina anormal con análogos de la hormona liberadora de gonadotropinas. Clin. Obstet. Gynecol. 1993; 3: 638-647.

21. Gannon MJ., Holt EM., Fairbank J et al. A randomised trial comparing endometrial resection and abdominal histerectomy for the treatment of hemorrhagia. BMJ 1991; 303: 1362-1365.

22. Comino R., Torrejon R. Resección endometrial en la hemorragia uterina disfuncional. Obst. y Gin. Latino-Americana 1993; 51(3): 305-324. 